\title{
Associations between \%AA (20:4 n6) and Relative Amounts of Other Body Fatty Acids
}

\author{
Arne Torbjørn Høstmark ${ }^{* *}$ and Anna Haug ${ }^{2}$
}

${ }^{1}$ Faculty of Medicine, Institute of Health and Society, University of Oslo, Norway, Box 1130 Blindern, 0318 Oslo, Norway, Telephone:+47 22844629.

${ }^{2}$ Department of Animal and Aquacultural Sciences, The Norwegian University of Life Sciences, Box 5003, 1432 Ås, Norway; Telephone:+47 67232664.

*Corresponding author: Arne Torbjørn Høstmark, Faculty of Medicine, Institute of Health and Society, University of Oslo, Norway.

Received date: February 10, 2020; Accepted date: February 18, 2020; Published date: February $26,2020$.

Citation: Arne T. Høstmark., Haug A, (2020) Associations between \%AA (20:4 n6) and Relative Amounts of Other Body Fatty Acids. J. Nutrition and Food Processing, 3(2); DOI:10.31579/2637-8914/024

Copyright: () 2020 Arne Torbjørn Høstmark, This is an open-access article distributed under the terms of the Creative Commons Attribution License, which permits unrestricted use, distribution, and reproduction in any medium, provided the original author and source are credited.

\section{Abstract:}

Body fatty acids are important in health and disease. Previously, we reported a positive association between \% AA (20:4 n6) and relative amounts of EPA (20:5 n3) and of some other fatty acids. We now study positive and negative correlations in general between \%AA and percentages of other fatty acids, as observed in chicken breast muscle. Two groups of fatty acids were identified: Group 1) with relative amounts correlating negatively with \%AA, and Group 2) with relative amounts correlating positively with \%AA. With the positive correlations, but not with the negative ones, we obtained similar scatterplots using true and random numbers. This apparent discrepancy is probably related to differences in skewness of the concentration distribution of some fatty acids. Most of Group 2 fatty acids are eicosanoid or docosanoid precursors. The overall correlation outcome may be largely explained by the particular concentration ranges of the fatty acids. We therefore suggest Distribution Dependent Correlations to be an evolutionary regulatory principle, possibly ensuring balance between various eicosanoids and docosanoids.

Keywords: arachidonic acid, eicosapentaenoic acid, docosahexaenoic acid, dihomo-gammalinolenic acid, eicosanoids, docosanoids, chickens, random numbers, muscle fat, chickens

\section{Definitions and abbreviations:}

Variability: the width or spread of a distribution, measured e.g. by the range and standard deviation.

Range: showing the highest and lowest values.

Distribution: graph showing the frequency distribution of a scale variable within a particular range. In this article, we also use distribution when referring to a particular range, $a-b$, on the scale.

Uniform distribution: every value within the range is equally likely. In this article, we may write "Distribution was from a to b", or "Distributions of $\mathrm{A}, \mathrm{B}$, and $\mathrm{C}$ were $\mathrm{a}-\mathrm{b}, \mathrm{c}-\mathrm{d}$, and $\mathrm{e}-\mathrm{f}$, respectively".

"Low-number variables" have low numbers relative to "high-number variables".

OA = Oleic Acid (18:1 c9); LA = Linoleic Acid (18:2 n6); ALA = Alpha Linolenic Acid (18:3 n3); AA = Arachidonic Acid (20:4 n6); EPA = Eicosapentaenoic Acid (20:5 n3); DPA = Docosapentaenoic Acid (22:5 n3); DHA = Docosahexaenoic Acid (22:6 n3); DGLA= dihomogammalinolenic acid (20:3 n6)

\section{Introduction}

In a diet trial in chickens, we previously showed that \%AA was positively associated with relative amounts of fatty acids serving as precursors of eicosanoids and docosanoids [1-3]. These latter compounds are important regulatory molecules in cell physiology. They are derived from polyunsaturated fatty acids with 20 or 22 carbon atoms, being formed in most organs and cell types, in reactions catalyzed by cyclooxygenases, lipoxygenases, and epoxygenases [4]. It is well known that EPA (20:5 n3) and AA (20:4 n6) are metabolic antagonists [5-7]. Eicosanoids derived from EPA may decrease inflammatory diseases [8-9], improve coronary heart diseases [10,11], and cancer [12], although a systematic Cochrane Review of selected studies questioned the beneficial effects of long-chain $\mathrm{n} 3$ fatty acids on all-cause and cardiovascular mortality [13].

When considering the beneficial health effects of foods rich in EPA, many of the positive effects would be anticipated if the fatty acid works to counteract effects of arachidonic acid (AA, 20:4 n6). This latter fatty acid is formed in the body from linoleic acid (LA, 18:2 n6), a major constituent in many plant oils, and is converted by cyclooxygenase and lipoxygenase into various eicosanoids, i.e. prostacyclin, thromboxane, and leukotrienes [5 - 6]. AA derived thromboxane $\mathrm{A}_{2}\left(\mathrm{TXA}_{2}\right)$ and leukotriene $\mathrm{B}_{4}\left(\mathrm{LTB}_{4}\right)$ have strong proinflammatory and prothrombotic properties $[5,6,12]$. Furthermore, endocannabinoids, which are derived from arachidonic acid, may have a role in adiposity and inflammation [14]. It has been reported that a decreased level of the serum EPA/AA ratio was a risk factor for cancer death in the general Japanese population [12]. It would appear, accordingly, that a coordinated regulation of the relative abundances of EPA and AA, and possibly also of other precursor fatty acids for the synthesis of eicosanoids and docosanoids, would be of physiological interest, so that an increase (decrease) in the percentage of one of these fatty acids would be accompanied by a concomitant increase (decrease) in percentage of many others. Indeed, we recently reported that 
that relative amounts of altogether 7 potential eicosanoid (docosanoid) precursor fatty acids were positively associated in breast muscle lipids of chickens [3].

Furthermore, we previously reported $[15,16]$ that \%AA is negatively associated with \%OA (oleic acid, 18:1 c9), indicating that relative amounts of AA may relate both positively and negatively to other fatty acid percentages. The aim of the present work was to examine - in general - positive and negative associations between \%AA and relative amounts of other fatty acids. Additionally, we wanted to elucidate whether the concentration ranges might be involved in the correlation outcomes.

\section{Materials and Methods}

\section{Chickens and diet}

We refer to our previous article [17] for details concerning the diet trial. In brief, from day 1 to 29 one-day-old Ross 308 broiler chickens from Samvirkekylling (Norway) were fed wheat-based diet containing $10 \mathrm{~g}$ fat per $100 \mathrm{~g}$ diet. ALA (18:3 $\mathrm{n} 3)$, a precursor of EPA, provided $15 \%$ of the fatty acids, and LA (18:2 n6), a precursor of AA, provided $21 \%$. The $\mathrm{n} 6 / \mathrm{n} 3$ ratio was 1.4 . Energy content of the feed was about $19 \mathrm{MJ} / \mathrm{kg}$. ALA provided $2.5 \%$ of the energy, and LA $4 \%$. Other components in the feed were: Histidine $0.1 \%$, choline chloride $0.13 \%$, mono-calcium phosphate $1.4 \%$, ground limestone $1.3 \%$, sodium chloride $0.25 \%$, sodium bicarbonate $0.2 \%$, vitamin A, E, D, K, B $0.18 \%$, L-lysine $0.4 \%$, DLmethionine $0.2 \%$, and L-threonine $0.2 \%$.

\section{Determination of fatty acids}

Fatty acid composition of breast muscle and feed was determined by gas chromatography in accordance with O'Fallon et al. [18].

\section{Calculations and statistical analysis}

Correlations. The following 22 fatty acids were determined in breast muscle lipids of the 163 chickens: $14: 0 ; 14: 1 \mathrm{c} 9 ; 15: 0 ; 16: 0 ; 16: 1 \mathrm{c} 9$; 17:0; 18:0; 18:1 t6,11; 18:1 c9; 18:2 c11; 18:2 n6; 20:0; 18:3 n6; 18:3 n3; 20:1 n9; 20:2 n6; 20:3 n6; 20:3 n3; 20:4 n6; 20:5 n3; 22:6 n3; and 22:5 $\mathrm{n} 3$. The sum (S) of all these fatty acids (SD), i.e. $8.85(2.62) \mathrm{g} / \mathrm{kg}$ wet weight $(n=163)$ was used in the denominator when calculating relative amounts of the fatty acids. For example, percentage arachidonic acid was computed as: $\% \mathrm{AA}=(\mathrm{AA} / \mathrm{S}) * 100$, and $\% \mathrm{EPA}=(\mathrm{EPA} / \mathrm{S}) * 100$. To obtain percentage amounts of other fatty acids, the calculation procedure was as shown for AA and EPA. With all fatty acids we computed correlation coefficients (Pearson's $r$ and/or Spearman's rho) to assess associations between the fatty acid percentages. We additionally made scatterplots to illustrate associations between relative amounts of these fatty acids.

\section{Calculations performed to possibly explain the correlation} outcome

\section{Range and variability.}

We computed ranges, mean values, and variabilities (coefficient of variation, $\mathrm{SD}$ ) of the fatty acids under investigation. For example, the range was $0.13-0.24 \mathrm{~g} / \mathrm{kg}$ for EPA, and $0.25-0.42 \mathrm{~g} / \mathrm{kg}$ for AA. To further examine concentration $(\mathrm{g} / \mathrm{kg})$ distributions of the various fatty acids, we made histograms; only some examples are shown.

Simplification.

To understand how associations between fatty acid percentages are brought about, we previously simplified the analyses by considering 3 variables only, i.e. the two fatty acids under investigation, and sum (R) of the remaining fatty acids. For example, $\mathrm{R}=\mathrm{S}-\mathrm{DPA}-\mathrm{DGLA}$, if DPA $(22: 5 \mathrm{n} 3)$ and DGLA (20:3 n6) are the fatty acids under investigation. Thus, $\% \mathrm{DPA}+\% \mathrm{DGLA}+\% \mathrm{R}=100$, or $\% \mathrm{DPA}=-\% \mathrm{DGLA}+(100-$ $\% \mathrm{R})$. With high \%R-values, this equation will approach \%DPA $=\%$ DGLA, showing a positive association between relative amounts of the variables; with a positive slope determined by the ranges of DPA and DGLA, as explained in more detail previously [2,3, 16, 18 - 20]. A similar reasoning is valid when considering the association between relative amounts of all other fatty acids. We present further details under Results and Discussion.

Are the correlation outcomes related to distributions of the fatty acids? A random number approach.

As reported previously, with AA and EPA the concentration distribution per se seems to be crucial for the correlation outcome between the fatty acid percentages [1,2]. If this conclusion is valid for the current analyses as well, we should anticipate similar correlation results with true and surrogate, random numbers for the fatty acids, if the random numbers were sampled with the true concentration ranges. Furthermore, the strength of the associations should be changed if we altered the distributions. We accordingly generated uniformly distributed RANDOM numbers with the physiological distributions of the couple of fatty acids under investigation, and of R. Since the diet trial had 163 birds, for each of the analyses below we generated 163 random numbers with the particular fatty acid distributions shown in Table 1. To clarify, we use upper case letters (RANDOM) or quotation marks in Figures or figure texts when working with random numbers.

Using random numbers in a previous computer experiment, we suggested [19] that, with 3 positive scale variables, two of which having low-number distribution, and low variability, as compared with the third variable, we might expect a positive association between relative amounts of the two low-number variables, and a negative association between percentage high-number variable and each of the low-number variable percentages. Furthermore, a decrease (increase) in the variability of either or both of the two low-number variables seemed to improve (make poorer) the association between their relative amounts. In contrast, a narrowing (broadening) of the distribution of the high-number variable seemed to make poorer (improve) the association between the two low-number variable percentages. In the present work, it seems that we have two lownumber variables (the pair of fatty acids under investigation) relative to a high-number variable (R). Therefore, the previous rules should apply for many of the current analyses. Thus, $\mathrm{R}$ is sum of the remaining fatty acids when omitting the two fatty acids under correlation analysis, i.e. $\mathrm{R}=\mathrm{S}-$ $\mathrm{A}-\mathrm{B}$, if $\mathrm{S}$ is the total sum of fatty acids, whereas $\mathrm{A}$ and $\mathrm{B}$ are the fatty acids under investigation. This means that $\% \mathrm{~A}+\% \mathrm{~B}+\% \mathrm{R}=100$, or $\% \mathrm{~B}$ $=-\% \mathrm{~A}+(100-\% \mathrm{R})$. Conceivably, $\mathrm{R}$ should be different for each of the calculations, since different pairs of fatty acids were used. It turned out that variation in $\mathrm{R}$ was small, due to great similarity between the ranges of the low-number fatty acids under investigation; $\mathrm{R}$ variability was generally $5-15 \mathrm{~g} / \mathrm{kg}$. We accordingly used the range $5-15$ for $\mathrm{R}$ in the computer experiments to investigate whether we might obtain the same correlation outcome with true values and random numbers, sampled with the true ranges. Additionally, by experimentally changing ranges with hypothetical values in computer experiments, we aimed at further clarifying whether the concentration ranges do govern the association between particular fatty acid percentages of the same sum. Since there are 
infinite many ways to change the distributions, we limit our analyses to narrowing or broadening of the physiological distributions. For each analysis, we made several repeats with new sets of random numbers; the general outcome of the repeats was always the same, but the correlation coefficients (Spearman's rho and/or Pearson's r), and scatterplots, varied slightly. We present the results as correlation coefficients, scatterplots, and regression analyses. SPSS 25.0 was used for the analyses, and for making figures. The significance level was set at $\mathrm{p}<0.05$. The experimental conditions are presented in more detail under "Results and Discussion".

\section{Results and Discussion}

\section{Descriptive data}

Minimum and maximum values, mean values $(\mathrm{g} / \mathrm{kg})$, with $\mathrm{SE}, \mathrm{SD}$, and mean percentage amounts (in descending order) of each of the 22 fatty acids are shown in Table 1 . Oleic acid was the main fatty acid providing $25 \%$ of all, with palmitic acid, linoleic acid and stearic acid on the next places, respectively.

\begin{tabular}{|c|c|c|c|c|c|c|}
\hline Fatty acid & Min & Max & Mean & SE & SD & \% \\
\hline $18: 1 \mathrm{c} 9$ & 1.04 & 8.56 & 2.44 & 0.09 & 1.07 & 24.9 \\
\hline $16: 0$ & 0.94 & 4.35 & 1.80 & 0.04 & 0.54 & 19.0 \\
\hline $18: 2 \mathrm{n} 6$ & 0.86 & 3.69 & 1.41 & 0.07 & 0.42 & 14.9 \\
\hline $18: 0$ & 0.65 & 1.70 & 0.89 & 0.01 & 0.16 & 9.6 \\
\hline $18: 3 \mathrm{n} 3$ & 0.12 & 2.40 & 0.53 & 0.03 & 0.32 & 5.2 \\
\hline $22: 5 \mathrm{n} 3$ & 0.21 & 0.43 & 0.31 & 0.00 & 0.04 & 3.5 \\
\hline $20: 4 \mathrm{n} 6$ & 0.25 & 0.42 & 0.31 & 0.00 & 0.03 & 3.4 \\
\hline $18: 2 \mathrm{c} 11$ & 0.14 & 0.52 & 0.24 & 0.00 & 0.05 & 2.6 \\
\hline $22: 6 \mathrm{n} 3$ & 0.11 & 0.32 & 0.19 & 0.00 & 0.04 & 2.3 \\
\hline $20: 5 \mathrm{n} 3$ & 0.13 & 0.24 & 0.18 & 0.00 & 0.02 & 2.0 \\
\hline $16: 1 \mathrm{c} 9$ & 0.03 & 0.78 & 0.18 & 0.01 & 0.11 & 1.8 \\
\hline $20: 3 \mathrm{n} 6$ & 0.06 & 0.11 & 0.08 & 0.00 & 0.01 & 0.8 \\
\hline $20: 1 \mathrm{n} 9$ & 0.00 & 0.32 & 0.08 & 0.00 & 0.09 & 0.5 \\
\hline $20: 3 \mathrm{n} 3$ & 0.04 & 0.09 & 0.05 & 0.00 & 0.01 & 0.5 \\
\hline $20: 2 \mathrm{n} 6$ & 0.04 & 0.06 & 0.05 & 0.00 & 0.01 & 0.4 \\
\hline $18: 1 \mathrm{t} 6,11$ & 0.01 & 0.15 & 0.04 & 0.00 & 0.02 & 0.4 \\
\hline $14: 0$ & 0.01 & 0.17 & 0.04 & 0.00 & 0.02 & 0.2 \\
\hline $17: 0$ & 0.01 & 0.07 & 0.02 & 0.00 & 0.01 & 0.2 \\
\hline $15: 0$ & 0.00 & 0.04 & 0.01 & 0.00 & 0.01 & 0.1 \\
\hline $18: 3 \mathrm{n} 6$ & 0.00 & 0.02 & 0.01 & 0.00 & 0.00 & 0.1 \\
\hline $14: 1 \mathrm{c} 9$ & 0.00 & 0.04 & 0.01 & 0.01 & 0.01 & 0.1 \\
\hline $20: 0$ & 0.00 & 0.02 & 0.01 & 0.00 & 0.00 & 0.1 \\
\hline
\end{tabular}

Note: some of the values appear as zero due to the number of decimals.

Table 1: Minimum and maximum values, mean values (g/ kg), with SE, SD, and \% of fatty acids in breast muscle lipids, in descending order of percentages; $\mathrm{n}=163$.
Correlation coefficients and scatterplots showing positive and negative correlations between \%AA and percentages of other fatty acids

We next investigated correlation coefficients and made scatterplots (Table 2, Figure $1-5$ ) to show associations between $\%$ AA and relative amounts of other fatty acids. We found 8 positive and 6 negative such correlations; however the positive scatterplot between \%AA and \%18:2 $\mathrm{c} 11$, as well as the negative ones with \%14:0, and \%14:1 c9 indicated poor separation (not shown). The remaining 7 positive associations between $\% \mathrm{AA}$ and relative amounts of other fatty acids are shown in Table 2 and Figures 1 - 3, left columns. Values of correlation coefficients are shown in Table 2. The results with Spearman's rho and Pearson's $r$ did not differ much, and all were with $\mathrm{p}<0.001, \mathrm{n}=163$.

\begin{tabular}{|c|c|c|}
\hline Fatty acid & Spearman's rho & Pearson's r \\
\hline $18: 1 \mathrm{c} 9$ & -0.928 & -0.935 \\
\hline $18: 3 \mathrm{n} 3$ & -0.902 & -0.910 \\
\hline $18: 0$ & +0.683 & +0.717 \\
\hline $22: 5 \mathrm{n} 3$ & +0.869 & +0.881 \\
\hline $22: 6 \mathrm{n} 3$ & +0.770 & +0.765 \\
\hline $16: 1 \mathrm{c} 9$ & -0.791 & -0.803 \\
\hline $20: 3 \mathrm{n} 6$ & +0.741 & +0.761 \\
\hline $20: 3 \mathrm{n} 3$ & +0.627 & +0.635 \\
\hline $20: 5 \mathrm{n} 3$ & +0.750 & +0.762 \\
\hline $20: 2 \mathrm{n} 6$ & +0.768 & +0.774 \\
\hline $18: 3 \mathrm{n} 6$ & -0.577 & -0.597 \\
\hline
\end{tabular}

Table 2: Correlation coefficients (Spearman's rho and Pearson's r) for associations between \%AA (20:4 n6) and relative amounts of other fatty acids in breast muscle lipids of chickens; all correlation coefficients are with $\mathrm{p}<0.001, \mathrm{n}=163$.

Scatterplots of \%AA vs. relative amounts of 20:5 n3; 20:2 n6; 22:5 n3; 20:3 n6; 18:0; 22:6 n3; and 20:3 n3, respectively were generally fair ( Figure 1-3, correlation coefficients are shown below the panels).

\section{Will we manage to mimic the true scatterplots using surrogate, random numbers for the fatty acids?}

Positive correlations with \%AA

We previously reported that we were able to largely reproduce some positive correlations between fatty acid percentages when using surrogate random numbers, sampled with the true ranges of the fatty acids [1-3]. We accordingly investigated whether we might obtain the positive correlations also with random numbers instead of true concentrations of the fatty acids investigated in this work. As shown in Figure 1 - 3, right columns, for all of the positive correlations between \%AA and percentages of other fatty acids, we did indeed find that also the 
RANDOM numbers, gave positive correlations that did not differ much from those obtained with the true values.

\section{Negative correlations with \%AA}

With negative correlations between \%AA and relative amounts of other fatty acids, the outcome was quite different from the positive ones: we did not manage to reproduce these correlations with random numbers (Figure
4 - 5, right columns). Rather, most of the negative correlations with the true values changed to become positive with random numbers, even though they were sampled with the true ranges of the fatty acids in question. The only exception was a poor negative association between \%"random number \%AA" and \%"random number 18:1 c9", in line with the outcome that we reported earlier [16].

\section{\# 1, POSITIVE associations between \%AA and percentages of other fatty acids}

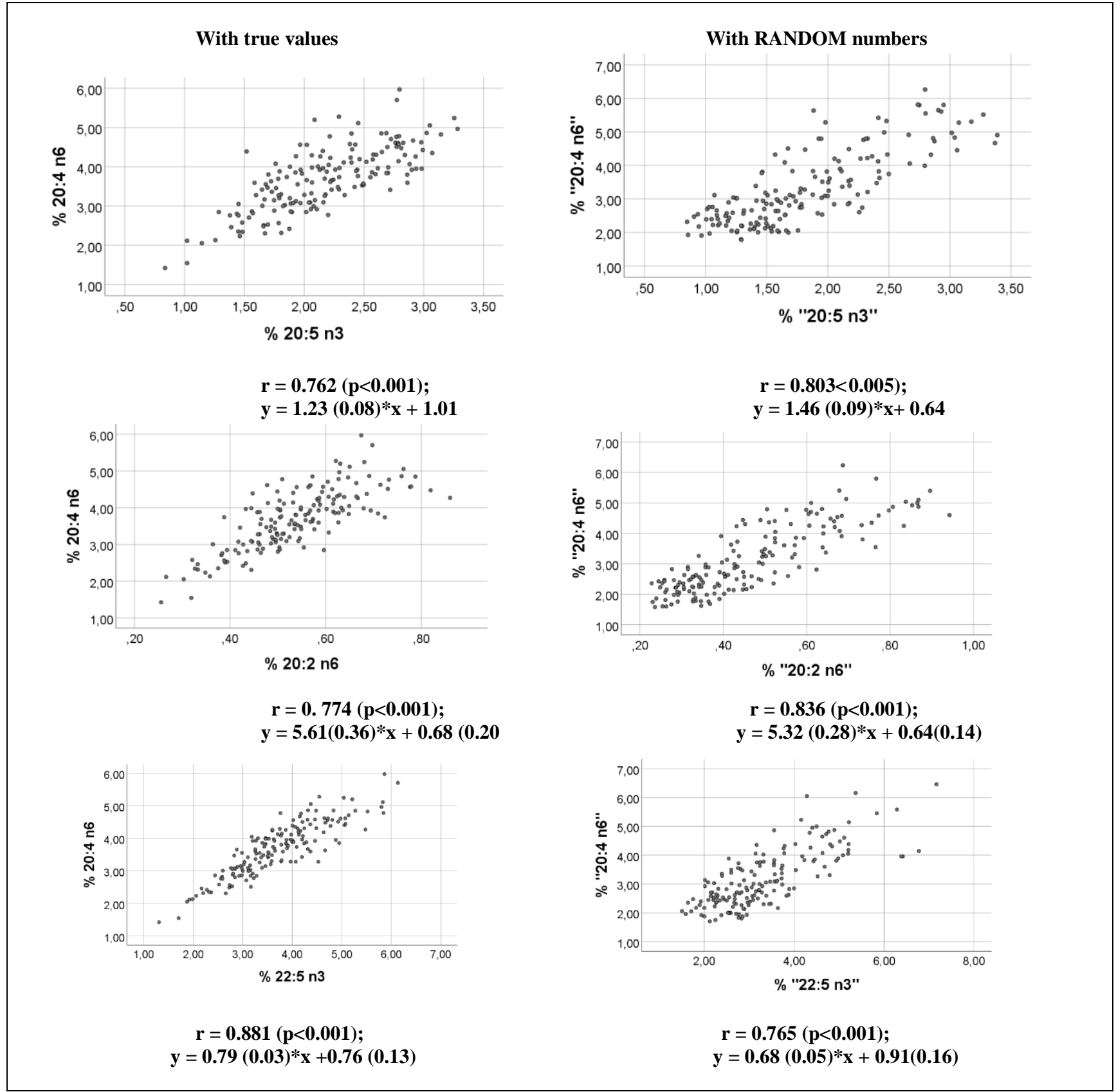

Figure 1. Scatterplots showing positive associations between percentage of arachidonic acid (20:4 n6) and of other fatty acids (left columns), and between $\%$ of random numbers representing 20:4 $\mathrm{n} 6$ and \% of other fatty acids (right columns; see Methods). Equations of the regression lines are shown below each of the panels. The general formula $y=a(S E){ }^{*} x+b(S E)$ is shown, where $y$ is the ordinate, and $x$ is the abscissa. Note that we use question marks with RANDOM numbers (right columns). 
\# 2, POSITIVE associations between \%AA and percentages of other fatty acids

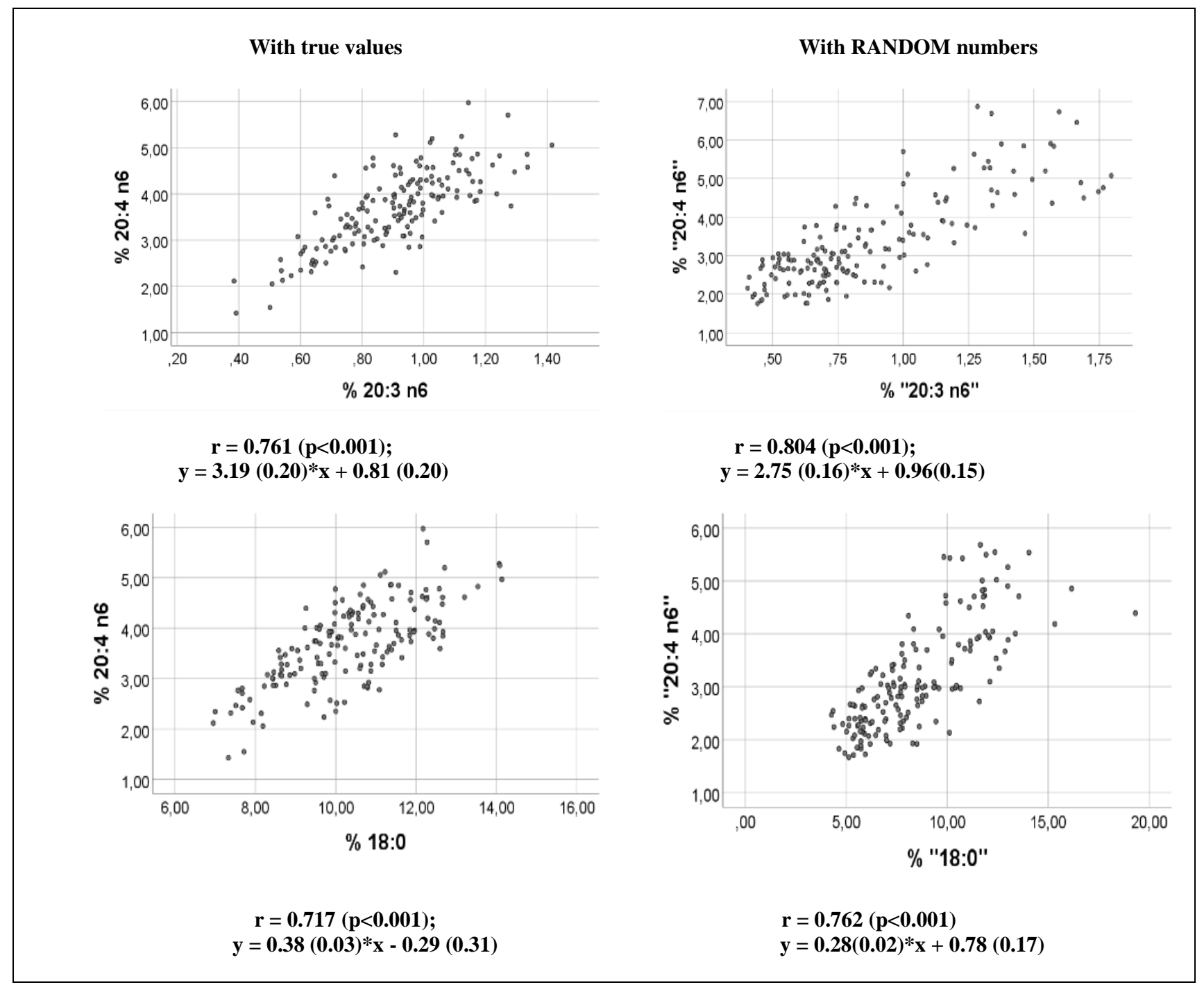

Figure 2. Scatterplots showing positive associations between percentage of arachidonic acid (20:4 n6) and of other fatty acids (left columns), and between $\%$ of random numbers representing 20:4 n6 and \% of other fatty acids (right columns; see Methods). Equations of the regression lines are shown below each of the panels. The general formula $y=a(S E){ }^{*} x+b(S E)$ is shown, where $y$ is the ordinate, and $x$ is the abscissa. Note that we use question marks with RANDOM numbers (right columns). 
\# 3, POSITIVE associations between \%AA and percentages of other fatty acids

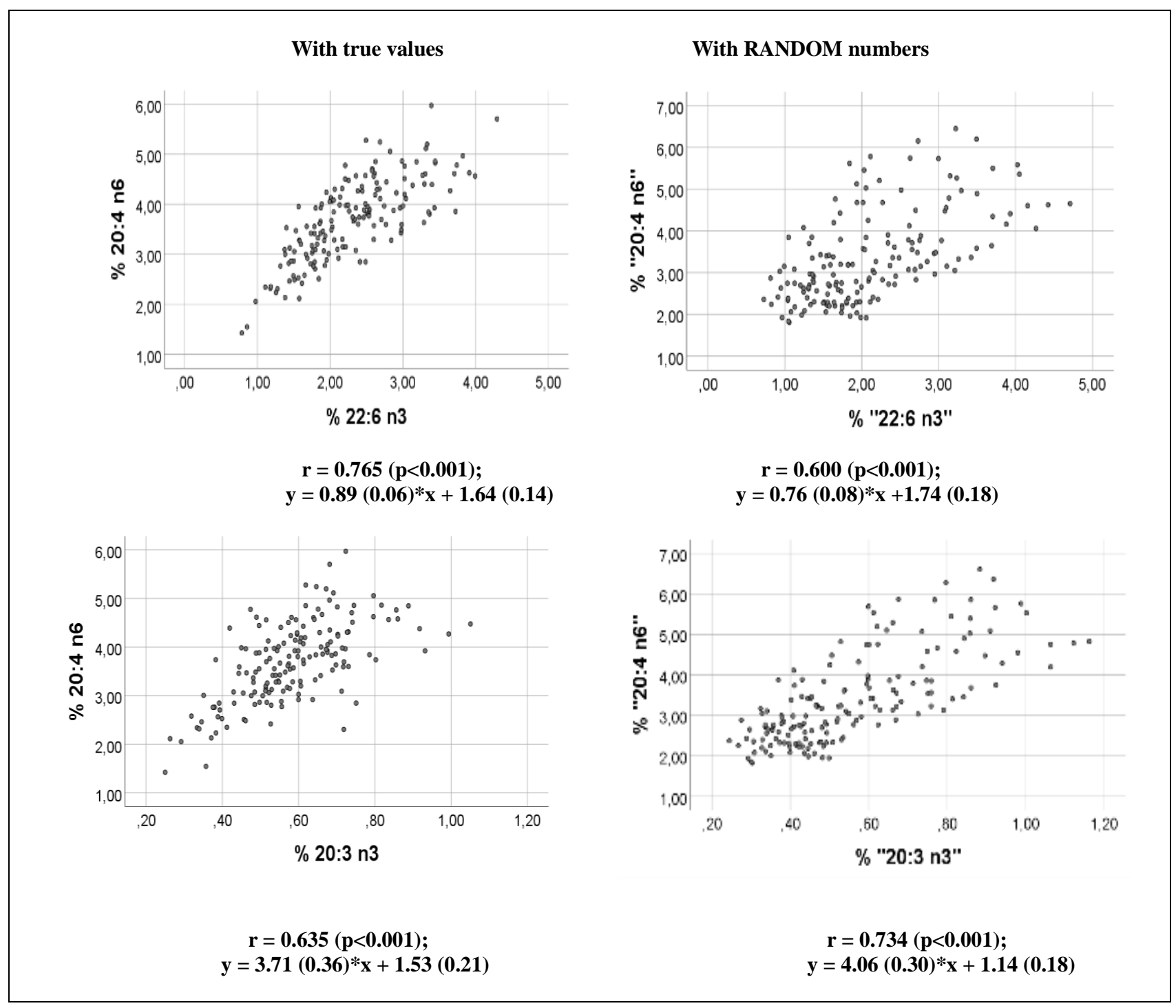

Figure 3. Scatterplots showing negative associations between percentage of arachidonic acid (20:4 n6) and of other fatty acids (left columns), and between \% of random numbers representing 20:4 n6 and \% of other fatty acids (right columns; see Methods). Equations of the regression lines are shown below each of the panels. The general formula $y=a(S E) * x+b(S E)$ is shown, where $y$ is the ordinate, and $x$ is the abscissa. Note that we use question marks with RANDOM numbers (right columns). 
\# 1, NEGATIVE associations between \%AA and percentages of other fatty acids

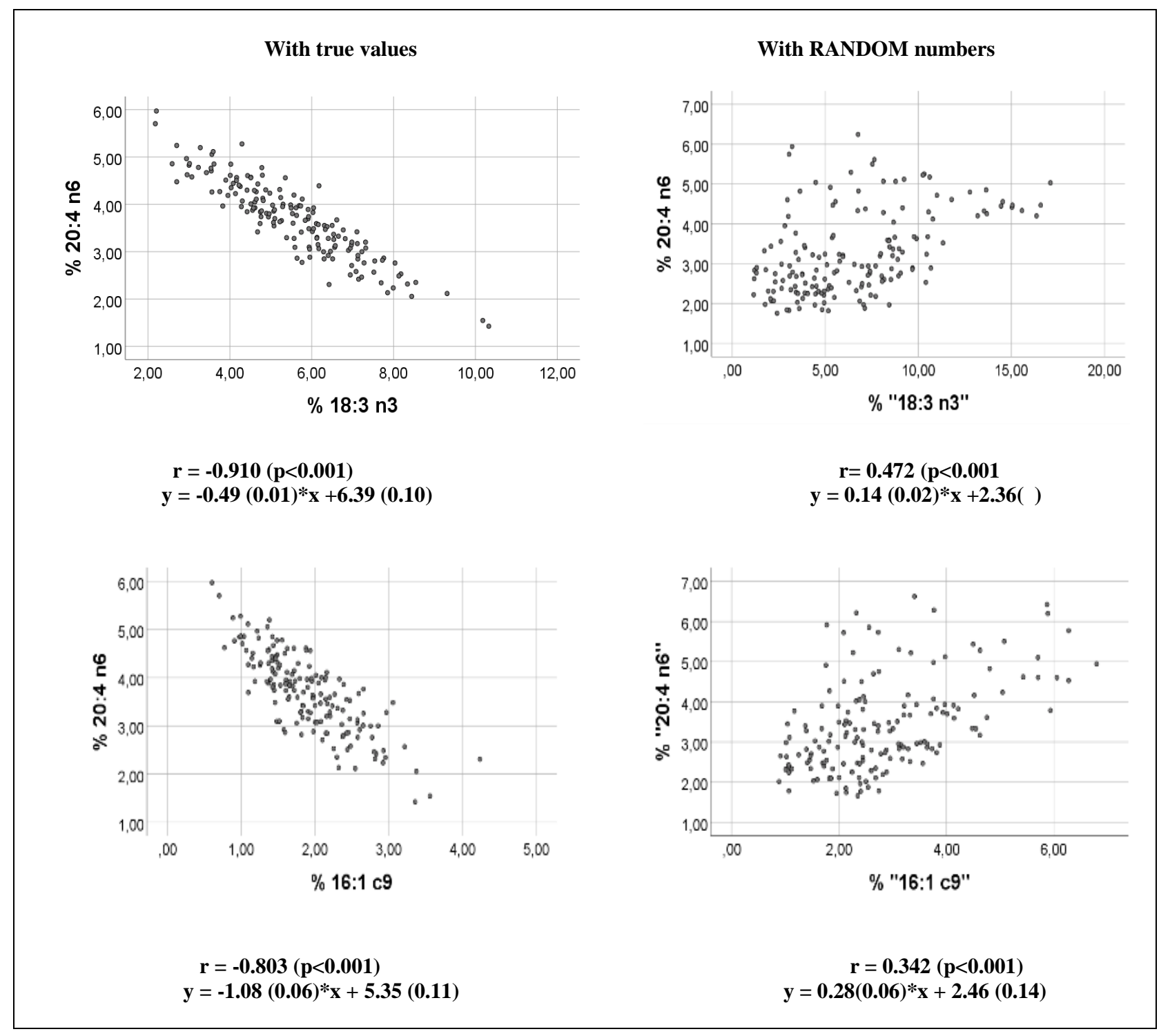

Figure 4. Scatterplots showing negative associations between percentage of arachidonic acid (20:4 n6) and of other fatty acids (left columns), and between \% of random numbers representing 20:4 n6 and \% of other fatty acids (right columns; see Methods). Equations of the regression lines are shown below each of the panels. The general formula $y=a(S E){ }^{*} x+b(S E)$ is shown, where $y$ is the ordinate, and $\mathrm{x}$ is the abscissa. Note that we use question marks with RANDOM numbers (right columns). 


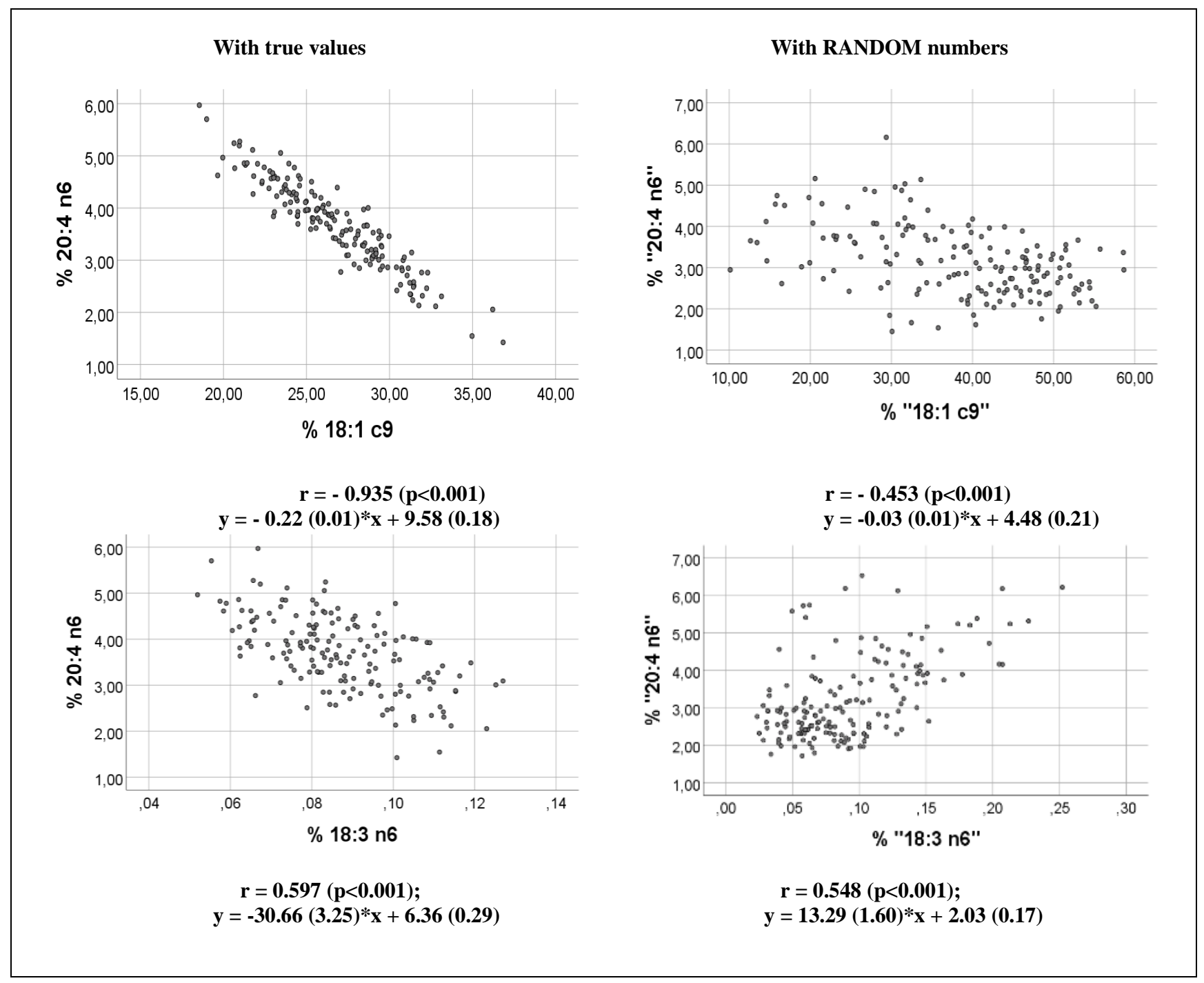

Figure 5. Scatterplots showing negative associations between percentage of arachidonic acid (20:4 n6) and of other fatty acids (left columns), and between $\%$ of random numbers representing 20:4 n6 and \% of other fatty acids (right columns; see Methods). Equations of the regression lines are shown below each of the panels. The general formula $y=a(S E){ }^{*} x+b(S E)$ is shown, where $y$ is the ordinate, and $x$ is the abscissa. Note that we use question marks with RANDOM numbers (right columns).

\section{Suggested explanation of the correlation outcome}

One interpretation of the present results could be that all of the positive associations between \%AA and percentages of other fatty acids are distribution-dependent ones, and that the negative ones are distribution- independent, i.e. not caused by the concentration distributions. This tentative conclusion might however be questioned, as discussed below.

To possibly clarify the apparent contradictory results observed with random numbers, we first consider in more detail some differences between the observed negative and positive correlations (Table 3). 


\begin{tabular}{|c|c|c|c|c|}
\hline Fatty acid & rho & Range & Max/Min & CV \\
\hline \multicolumn{5}{|c|}{ Negative correlations with \%20:4 n6 } \\
\hline $18: 3 \mathrm{n} 3$ & -0.902 & $0.12-2.40$ & 20.0 & 60.4 \\
\hline $18: 1 \mathrm{c} 9$ & -0.928 & $1.04-8.56$ & 8.2 & 43.9 \\
\hline $16: 1$ & -0.791 & $0.03-0.78$ & 26.0 & 61.1 \\
\hline $18: 3 \mathrm{n} 6$ & -0.577 & $0.00-0.02$ & 7.3 & 44.8 \\
\hline \multicolumn{5}{|c|}{ Positive correlations with \%20:4 n6 } \\
\hline $20: 3 \mathrm{n} 6$ & +0.741 & $0.06-0.11$ & 1.8 & 11.0 \\
\hline $20: 3 \mathrm{n} 3$ & +0.627 & $0.04-0.09$ & 2.3 & 12.2 \\
\hline $20: 2 \mathrm{n} 6$ & +0.768 & $0.04-0.06$ & 1.5 & 13.7 \\
\hline $18: 0$ & +0.683 & $0.65-1.70$ & 2.6 & 18.7 \\
\hline $22: 5 \mathrm{n} 3$ & +0.869 & $0.21-0.43$ & 2.0 & 13.2 \\
\hline $20: 4 \mathrm{n} 6$ & +1.000 & $0.25-0.42$ & 1.7 & 9.4 \\
\hline $22: 6 \mathrm{n} 3$ & +0.770 & $0.11-0.32$ & 2.9 & 21.2 \\
\hline $20: 5 \mathrm{n} 3$ & +0.750 & $0.13-0.24$ & 1.8 & 11.7 \\
\hline
\end{tabular}

Note: some values appear as zero due to the number of decimals; more decimals were included when calculating max/min values.

Table 3. Spearman's rho, range, $\mathrm{max} / \mathrm{min}$, and coefficient of variation (CV) of negative and positive associations between \%AA (20:4 n6) and relative amounts of other fatty acids. All rho-values are with $\mathrm{p}<0.001, \mathrm{n}=163$.

It turned out that variability was much higher for the negative than for the positive correlations.

\section{Is skewness of distributions involved to explain the} correlations?

We previously reported that correlations between percentages were
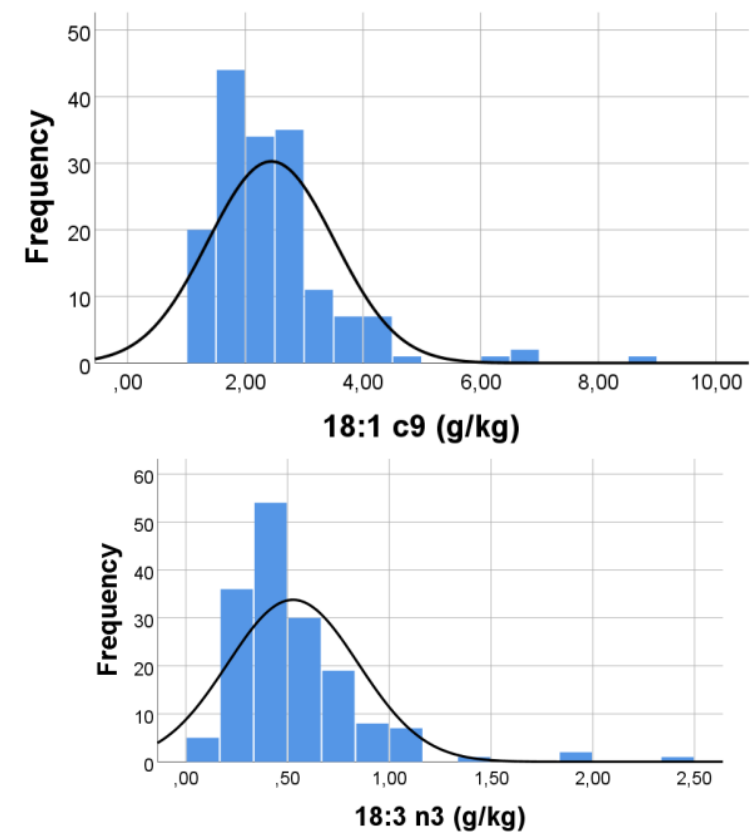

largely dependent upon the concentration distributions [1, 2, 19], as well as skewness [21]. In the present study we found high positive skewness of the absolute values of fatty acids with percentages correlating negatively with \%AA (Figure 6).
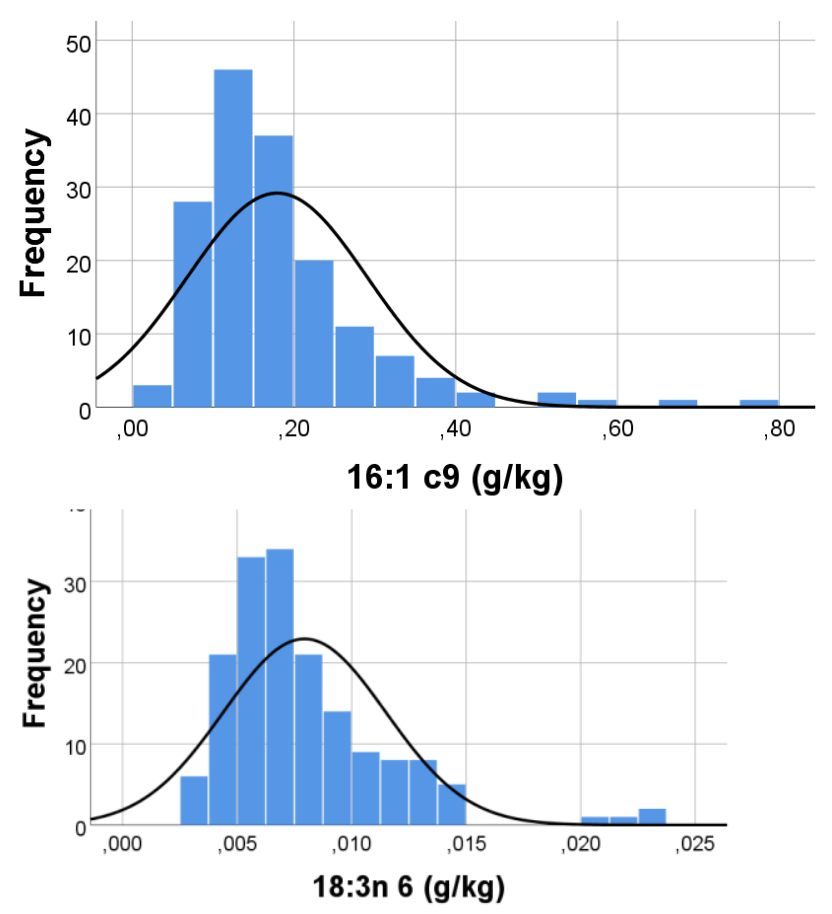

Figure 6. Skewness of histograms of absolute values of 18:1 c9, 16:1, 18:3 n3, and 18:3 n6. Skewness values of 18:1 c9, 16:1, 18:3 n3, and 18:3 n6, respectively, were: $2.268,2.270,2.502$, and 1.78 . 
Skewness of histograms of absolute values of 18:1 c9, 16:1, 18:3 n3, and 18:3 n6 were $2.268,2.27,2.50$, and 1.78, respectively (Figure 6). In contrast to this, we found minor skewness values for fatty acids with relative amounts correlating positively with \%AA; skewness values (in parentheses) were: $20: 3 \mathrm{n} 6$ (0.70); 20:4 n6 (0.66); 20:5 n3 (-0.16); 22:5 n3 (0.52); 22:6 n3 (0.76); 20:3 n3 (1.06); 20:2 n6 (0.46); histograms not shown.

\section{How would skewness influence correlations, as found with true values and random numbers?}

Skewness could be a significant factor to consider when we use substitute, random numbers to replace the true values of the fatty acids. The reason is that we did not manage to generate random numbers with distributions similar to those of the true values. Instead, we generated uniformly distributed random numbers ("rectangular distributions") within the true ranges of the fatty acids. Thus, since the true histograms had a tail to the right side (positive skewness), then we would obtain more of high numbers than was intended, if replacing them with uniformly distributed random numbers. Below we will present some considerations of how our substitute, random numbers might influence correlations between percentages.

If we utilize the previous algebraic approach [1-3,16,19], with 3 variables $(\mathrm{A}, \mathrm{B}, \mathrm{C})$, i.e. $\% \mathrm{~A}+\% \mathrm{~B}+\% \mathrm{C}=100$, or $\% \mathrm{~B}=-\% \mathrm{~A}+(100-\% \mathrm{C})$, we see that the expression $(100-\% \mathrm{C})$ will be increasingly smaller with increasing $\% \mathrm{C}$-values. This is a situation promoting a positive association between $\% \mathrm{~A}$ and $\% \mathrm{~B}$, as explained previously $[1,3,19]$. However, also with decreasing $\% \mathrm{~A}$ and $\% \mathrm{~B}$ values we should obtain increased levels of $\% \mathrm{C}$, since the sum of $\mathrm{A}, \mathrm{B}$, and $\mathrm{C}$ percentages is always $100 \%$. Thus, a situation with increased $\% \mathrm{C}$ values, and/or decreased $\% \mathrm{~A}(\mathrm{~B})$ should be in favour of a positive $\% \mathrm{~A}$ vs. $\% \mathrm{~B}$ association, rather than a negative one. Conversely, low values of $\% \mathrm{C}$, eventually as a result of high $\% \mathrm{~A}(\mathrm{~B})$, should favour a negative $\% \mathrm{~A}$ vs. $\% \mathrm{~B}$ correlation.

Example 1. We consider the true negative association between \% palmitoleic acid (PA, 16:1) and \% AA (20:4 n6). We first compute R, which is sum of the remaining fatty acid when omitting the two under investigation). Thus, $\% \mathrm{PA}+\% \mathrm{AA}+\% \mathrm{R}=100$, or $\% \mathrm{AA}=-\% \mathrm{PA}+(100$ $-\% \mathrm{R})$. In this case, we found that both $\mathrm{PA}$ and $\mathrm{R}$ had large positive skewness (2.26, and 2, 22, respectively), whereas AA had minor skewness (0.66). Thus, if we replace the highly skewed, true $\mathrm{R}$ and PA values with random numbers having uniform (rectangular) distribution, then we would have more of higher-than-intended values of both PA and R. High R-values should favour a positive \%AA vs. \%PA association, whereas high PA values should have the opposite effect. This reasoning raises the question of which of these effects will prevail. Since R has much higher values $($ range $5.1-22.1$; mean $=8.37)$ than PA $(0.03-0.78$; mean $=0.18)$, the skewness effect upon the \%AA vs. \%PA correlation should be dominated by the R-skewness. This means that we should expect a movement towards a positive correlation between \%AA and \%PA when replacing the highly skewed true $\mathrm{R}$ and $\mathrm{PA}$ values with random numbers having uniform distribution; this was indeed the correlation outcome shown in Figure 5, i.e. a change from negative to positive ones. A similar reasoning may be done for other fatty acids with large skewness. The minor skewness of AA should probably not have any major effect on the $\%$ AA vs \%PA association.
Example 2. We previously reported [16] a weak negative association between \%OA (18:1 c9) and \%AA, also with uniformly distributed random numbers instead of the true ones for OA, AA, and R. A similar outcome was found in the current repeat, with a new set of random numbers (i.e. rho $=-0.464, \mathrm{p}<0.001$, for the \%OA vs. \%AA association, $\mathrm{n}=163$ ). But how should we explain that the negative correlation outcome prevailed, albeit poorer, also with random numbers in this case? The explanation might be found when considering magnitudes and skewness of OA, AA, and R. Their ranges were 1.04-8.56; $0.25-0.42$; and $4.02-14.35$, respectively. Corresponding skewness vales were 2.27, 0.66 , and 2.17. Thus, OA - as well as R - were high-number variables with high positive skewness. We next utilize the equation $\% \mathrm{AA}=-\% \mathrm{OA}+$ $(100-\mathrm{R})$. As explained above, we should expect a movement towards a positive (negative) correlation between $\% \mathrm{AA}$ and $\mathrm{OA}$ in response to increasing (decreasing) the $\mathrm{R}(\% \mathrm{R})$ values. When replacing the highly positive skewed distributions of $\mathrm{R}$ with random numbers having rectangular distribution, we should move the R-distribution towards higher values, thereby favouring a positive \%AA vs. \%OA association. However, an opposite effect is anticipated when replacing the strong positive OA distribution with uniformly distributed random numbers. Since $\% \mathrm{OA}+\% \mathrm{AA}+\% \mathrm{R}=100$, an increase in $\% \mathrm{OA}$ - caused by the random number replacement - must be accompanied by a decrease in $\% \mathrm{R}$, an effect that should favour a negative \%AA vs. \%OA association. Accordingly, when replacing true values with the random numbers, then the final results could well be a poorer negative \%AA vs. \%OA association, since the random number $\%$ R-distribution had slightly more of high $\% \mathrm{R}$-values as compared with the true distribution (upper quartiles of $\% \mathrm{R}$ being 76.1 and $71.6 \%$, respectively). However, these results do not clarify to what extent the nice negative \%AA vs. \%OA scatterplot obtained with true values might be explained by distributions per se of $\mathrm{OA}$ and AA. Nevertheless, with negative correlations between fatty acid percentages, we should probably not expect the same outcomes with true values and uniformly distributed random numbers, due to high skewness of absolute values.

In our previous and present analyses of distribution dependent correlations, we have suggested 3 ways to possibly predict the direction and strength of such associations: Our first approach [1, 2, 19] was to utilize the equation $\% \mathrm{~B}=-\% \mathrm{~A}+(100-\% \mathrm{C})$. Next we considered skewness of the histograms of relative amounts of the variables [21]. We also showed a geometric approach [19]. The present results suggest that high positive skewness of the distribution of absolute amounts of some fatty acids might -at least partly -explain why we generally did not succeed in reproducing negative correlations when using uniformly distributed random numbers.

\section{Are distributions always a major point?}

Our previous and present experiments with random numbers raise the of whether the concentration distribution per se of fatty acids in general governs whether their relative amounts are positively or negatively associated, or not related at all. That range is essential for such correlations seems to be well accounted for concerning positive correlations between fatty acid percentages. However, with negative ones we did not manage to reproduce the true scatterplots with random numbers. Our lack of success in that regard could be related to the high positive skewness of the distributions encountered with fatty acids having 
percentages that correlated negatively with \%AA. However, to what extent concentration ranges also govern the negative correlations does not seem to be fully clarified by the present results; therefore, we cannot rule out the possibility that other mechanisms might be involved as well.

Nevertheless, the results raise the intriguing question of whether evolution might have "chosen" particular concentration ranges for each of the many types of fatty acids, to ensure that percentages of some of them must be positively associated whereas others possibly might be negatively correlated. Our finding that relative amounts of fatty acids serving as precursors for eicosanoids and docosanoids correlated positively could be an evolutionary mechanism ensuring a proper balance between molecules with opposing actions.

\section{Do the findings have health implications?}

Eicosanoids and docosanoids are molecules with strong physiological effects [4]. It seems reasonable to suggest that the observed association between relative amounts of their fatty acid precursors might ensure a proper balance between physiological effects of eicosanoids and docosanoids. For example, the thromboembolic risk should decrease by increasing the EPA percentage and decreasing \%AA in platelet phospholipids, thereby decreasing the production of $\mathrm{TXA}_{2}$ and platelet aggregation. In keeping with this suggestion, it has been reported that platelet signaling responses are modified by EPA and DHA [22].

From the present results it may be hypothesized that a disturbance in Distribution Dependent Correlations so that the positive association between some fatty acids percentages would be disturbed, and perhaps even lost, could increase the risk of e.g. AA related conditions and diseases, but we do not know have data to corroborate this hypothesis. We do not have an obvious, general physiological explanation for the negative correlations between \%AA and other fatty acid percentages. However, precursor-product relationships involving feed-back regulation could be involved, as we previously suggested [16]. Furthermore, increased supply of oleic acid might reduce AA percentage by pure mass action. Additionally, inverse regulation between OA and AA could be effected through an inhibition by AA of Delta- 9 desaturase, thereby decreasing the OA percentage; previous studies suggest that this mechanism might take place [23].

\section{Limitations of the study}

This work was confined to studying the association between relative amounts of selected fatty acids in chicken muscle. We do not know to what extent the suggested phenomenon of Distribution dependent correlations is valid for other fatty acids as well. Furthermore, the analyses were based upon fatty acids found in breast muscle lipids of chickens, and we do not know the generalizability of our results, as related to different organs, tissues or compartments, and to various species, including man. Furthermore, we do not know whether the balance between various eicosanoids and docosanoids might be influenced by diet, drugs and other external factors. Future work in this field should include studies to explore whether the fatty acid distribution might also govern the association between relative amounts of other fatty acids. Comparable studies should be done in various species, including man, and modifying factors should be investigated.

\section{Authors' contributions}

This work is a spin-off study of a previously published diet trial, conceived and carried out by AH. ATH conceived the present study, did the calculations, statistical analyses, and wrote the article. Both authors participated sufficiently - intellectually or practically - in the work, to take public responsibility for the content of the article. Both authors read and approved the final manuscript.

\section{Ethics approval}

The diet trial in chickens was performed in accordance with National and international guidelines concerning the use of animals in research (Norwegian Animal and Welfare Act, European Convention for the protection of Vertebrate Animals used for Experimental and other Scientific Purposes, CETS No.: 123 1986). The Regional Norwegian Ethics Committee approved the trial, and the experimental research followed internationally recognized guidelines. There are no competing interests.

\section{Conclusions}

The present analyses show that some fatty acid percentages may be positively associated, whereas others are negatively correlated. The positive associations - but not the negative ones - seem to be well explained by the distribution per se of the fatty acid concentrations, i.e. they are Distribution Dependent Correlations. Positive correlations between fatty acid percentages could serve to balance effects of various eicosanoids and docosanoids. We speculate whether a disturbance in Distribution Dependent Correlations could increase the risk of AA related conditions and diseases.

\section{Acknowledgements}

We thank the collaborators at the Norwegian University of Life Sciences, and at the Animal Production Experimental Center, Ås, Norway, especially Nicole F. Nyquist, Therese Mosti and Malin Andersen.

\section{Funding}

The study of which the present one is a spin-off, was funded by grant no 190399 from the Norwegian Research Council, and Animalia; the Norwegian Meat and Poultry Research Centre.

\section{References}

1. Høstmark AT, Haug A (2018) The Fatty Acid Distribution per se Explains Why Percentages of Eicosapentaenoic Acid (20:5 n3) and Arachidonic Acid (20:4 n6) are Positively Associated; a Novel Regulatory Mechanism? J Nutr Diet Suppl 2(1): 103

2. Høstmark AT, Haug A (2019) Associations Between \%AA (20:4 n6) and Percentages of EPA (20:5 n3), DPA (22:5 n3), and DHA (22:6 n3) Are Distribution Dependent in Breast Muscle Lipids of Chickens. J Nutr Diet Suppl 3(1): 103

3. Høstmark AT., Haug A., (2020) Relative Amounts of Eicosanoid and Docosanoid Precursor Fatty Acids Are Positively Associated: A Distribution Dependent Regulation. J Nutr Food Proc, 3(1);

4. Christie W. W. The Lipid Web (2019) Eicosanoids and Related Oxylipins, an Introduction 
5. Mayes PA (2000) Metabolism of unsaturated fatty acids and eicosanoids. In: Murray RK, Granner DK, Mayes PA, Rodwell VW, ed. Harper's Biochemistry, New York: McGraw-Hill, p 250-258

6. Baker RR. The eicosanoids: a historical overview. 1990 Clin Biochem. Oct. 23(5):455-8

7. Gogus U, Smith C (2010) n-3 Omega fatty acids: a review of current knowledge. Int J Food Sci Tech 45:417-436

8. Kremer JM, Bigauoette J, Michalek AV (1985) Effects of manipulation of dietary fatty acids on manifestations of rheumatoid arthritis. Lancet: $184-187$

9. Lorenz R, Weber PC, Szimnau P (1989) Supplementation with n-3 fatty acids from fish oil in chronic inflammatory bowel disease - a randomized, placebo-controlled, double blind crossover trial. J Intern Med Suppl 731: 225-732

10. Kromhout D. (2012) Omega-3 fatty acids and coronary heart disease the final verdict? Curr Opin Lipidol 23:554-559

11. Watanabe T, Ando K, Daidoji H, Otaki Y, Sugawara S, et al. (2017) A randomized controlled trial of eicosapentaenoic acid in patients with coronary heart disease on statins: The CHERRY study. J Cardiol 70:537-544

12. Nagata M, Hata J, Hirakawa Y, Mukai N, Yoshida D, et al. (2017) The ratio of serum eicosapentaenoic acid to arachidonic acid and risk of cancer death in a Japanese community: The Hisayama Study. J Epidemiol 27: 578-583

13. Abdelhamid AS, Brown TJ, Brainard JS, Biswas P, Thorpe GC, et al. (2018) Omega-3 fatty acids for the primary and secondary prevention of cardiovascular disease. Cochrane Database of Systematic Reviews.

14. Alvheim AR, Malde MK, Osei-Hyiaman D, Lin YH, Pawlosky RJ, et al. (2012) Dietary linoleic acid elevates endogenous 2-AG and anandamide and induces obesity. Obesity 20:1984-1994
15. Haug A, Olesen I, Christophersen OA (2010) Individual variation and intraclass correlation in arachidonic acid and eicosapentaenoic acid in chicken muscle. Lipids Health Dis $15: 37$

16. Høstmark AT, Haug A (2019) The inverse association between relative abundances of oleic acid and arachidonic acid: a case of distribution dependent regulation? Lipids Health Dis 18:123

17. Nyquist NF, Rødbotten R, Thomassen M, Haug A (2013) Chicken meat nutritional value when feeding red palm oil, palm oil or rendered animal fat in combinations with linseed oil, rapeseed oil and two levels of selenium. Lipids Health Dis 12:69

18. O'Fallon JV, Busboom JR, Nelson ML, Gaskins CT (2007) A direct method for fatty acid methyl ester synthesis: application to wet meat tissues, oils, and feedstuffs. J Anim Sci 85:1511-1521. doi: 10.2527/jas.2006-491

19. Høstmark AT (2019) Association between Percentages of Scale Variables, as Related to Distributions. J Nutr Diet Suppl 3(1): 104

20. Høstmark AT, Haug A (2019) High Variability of Oleic Acid (OA, 18:1 c9) improves the Positive Association between \%EPA (20:5 n3) and \%AA (20:4 n6). J Nutr Diet Suppl 3(1): 106

21. Høstmark AT (2019) Body Fatty Acids, Nutrition, and Health: Is Skewness of Distributions a Mediator of Correlations? J Nutr Food Proc 2(1);

22. McCue B, Shaull L, Kollasch A, Ye S, Whiteheart S, et al. (2014) The effect of EPA and DHA on platelets treated with multiple agonists found in the thrombus core FASEB J 28, 1. Suppl

23. Miyazaki M, Ntambi JM (2008) Fatty acid desaturation and chain elongation in mammals. In: Vance DE, Vance JE, ed. Biochemistry of Lipids and Membranes, UK: Elsevier, p 191211
This work is licensed under Creative Commons Attribution 4.0 License

To Submit Your Article Click Here: Submit Manuscript

DOI: $10.31579 / 2637-8914 / 024$

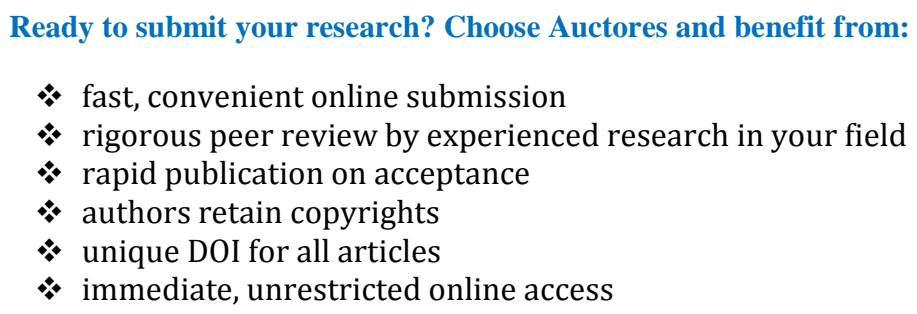

Ready to submit your research? Choose Auctores and benefit from:

* fast, convenient online submission

* rigorous peer review by experienced research in your field

* rapid publication on acceptance

* authors retain copyrights

* unique DOI for all articles

* immediate, unrestricted online access

At Auctores, research is always in progress.

Learn more www.auctoresonline.org/journals/nutrition-and-foodprocessing 